\title{
Channel estimation technique with SNR measurement by minimum accumulated distance of Viterbi decoder
}

\author{
Ki-O Song and Hyeok-Koo Jung ${ }^{\text {a) }}$ \\ Hanbat National University, \\ San 16-1, Dukmyung-Dong, Yuseong-Gu, Daejeon, 305-719, Korea \\ a) junghk@hanbat.ac.kr
}

\begin{abstract}
This paper addresses channel estimation technique with SNR measurements for low SNRs. The LS estimator is simple and adequate for high SNRs, but the LMMSE estimator of assuming a priori knowledge of SNR value and channel covariance has a better gain over LS estimator for low SNRs. In this paper we propose an SNR measurement method with a little complexity by using minimum accumulated distance in Viterbi decoder. The measured SNR value is used in the LMMSE estimator and the performance is presented both in terms of mean square error and bit error rate compared to the LS estimator and the perfect channel state information case.
\end{abstract}

Keywords: OFDM, channel estimation, LMMSE, Viterbi decoder, Minimum Accumulated Distance

Classification: Science and engineering for electronics

\section{References}

[1] IEEE Std 802.11a-1999, Part 11, Wireless LAN Medium Access Control (MAC) and Physical Layer (PHY) specifications: High-speed Physical Layer in the $5 \mathrm{GHz}$ Band.

[2] IEEE P802.11n ${ }^{\mathrm{TM}} / \mathrm{D} 3.00$, Part 11, Wireless LAN Medium Access Control (MAC) and Physical Layer (PHY) specifications.

[3] J. Heiskala and J. Terry, OFDM Wireless LANs, A Theoretical and Practical Guide, SAMS, 2001.

[4] Louis L. Scharf, Statistical Signal Processing, Addison-Wesley, 1991.

[5] D. R. Pauluzzi and N. C. Beaulieu, "A Comparison of SNR Estimation Techniques for the AWGN Channel," IEEE Trans. Commun., vol. 48, pp. 1681-1691, Oct. 2000.

[6] H. Xu, G. Wei, and J. Zhu, "A Novel SNR Estimation Algorithm for OFDM," IEEE VTC 2005-spring, vol. 5, pp. 3068-3071, June 2005.

[7] J.-J. van de Beek, O. Edfors, M. Sandell, S. K. Wilson, and P. O. Borjesson, "On channel estimation in OFDM systems," IEEE VTC '95, vol. 2, pp. 815-819, July 1995.

[8] S. Haykin, Adaptive Filter Theory, Prentice-Hall Inc., 1996. 


\section{Introduction}

Recently, orthogonal frequency-division multiplexing (OFDM) system has been known to be effective for mobile broadband radio transmission, but its receiver complexity prohibits real implementation at a mobile terminal. In wireless LAN systems like IEEE801.11a standard [1] and IEEE801.11n standard [2], its receiver performance depends on channel estimation technique which has to be implemented in a receiver. The least squares (LS) algorithm is a famous channel estimation technique [3] because of its simple and adequate implementation in a receiver for high SNRs, but it has a drawback of poor performance for low SNRs. While the linear minimum mean square error (LMMSE) [4] has been known to have a better performance rather than LS algorithm for low SNRs, but it needs some parameters like autocovariance matrix of channel and signal-to-noise ratio (SNR) value. Several SNR estimation techniques have been proposed for AWGN channel [5] and mobile environments of time variant channel [6] why minimum mean square error (MMSE) algorithm needs SNR estimates. There is a previous SNR estimation methodology by minimum accumulated distance (MAD) in Viterbi decoder [3], so we expand it as 3 bit soft decision Viterbi decoder which is common in OFDM systems for HIPERLAN/2 channel model for a distinct separation between probability density functions (PDF) of MAD per SNR. In this new approach, it is not necessary to use additional hardware for SNR estimation unlike the above mentioned SNR estimation algorithms. These SNR estimates are used in the LMMSE channel estimation algorithm, which results in a close performance as the ideal channel case.

In this paper we propose a hybrid algorithm that combines SNR estimation technique using Viterbi decoder and the LMMSE channel estimation algorithm, and simulate in HIPERLAN/2 channel environments and show the results. In simulation section we evaluate the proposed channel estimator by simulating 16-QAM signaling scheme. The performance is presented both in terms of mean square error (MSE) and bit error rate (BER).

\section{Hybrid SNR estimation and the LMMSE channel estimation technique}

In this section for the reduction of the implementation complexity and a hybrid SNR estimation and the LMMSE channel estimation technique is proposed, which is shown in Fig. 1. In the transmitter, there is an $1 / 2$ rate convolutional encoder, and in the receiver there is a Viterbi decoder which is used for SNR estimation. SNR estimation is not necessary for the LS algorithm, but necessary for the LMMSE algorithm, SNR estimation block in Fig. 1 is only used for the LMMSE case.

\subsection{SNR estimation using Viterbi decoder in HIPERLAN/2 chan- nels}

Viterbi decoder is a solution for convolutional encoder and it has a good error correction capability against burst errors, so it is widely used in many 


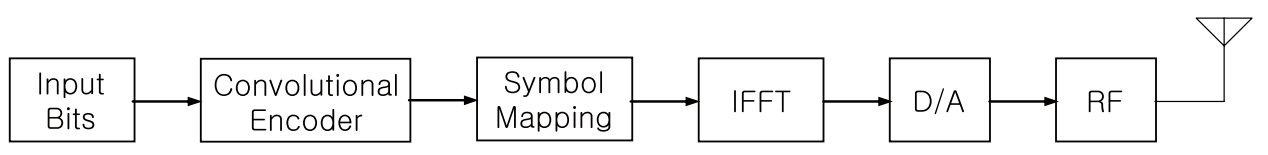

(a) Transmitter

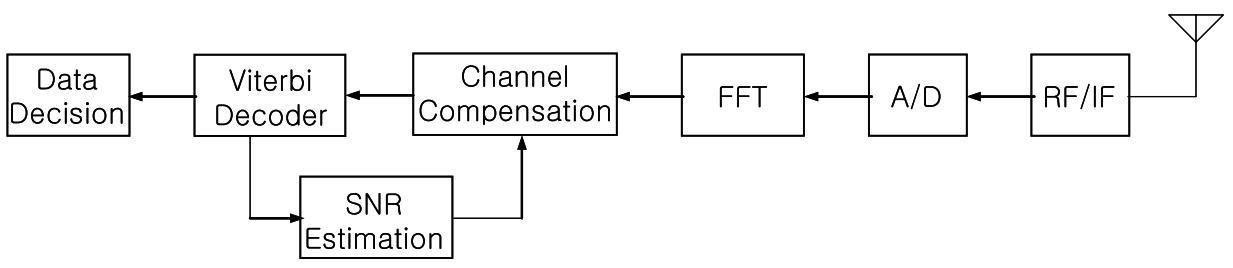

(b) Receiver

Fig. 1. Block diagram of the proposed algorithm

wireless LAN standards as IEEE802.11a and IEEE802.11n. In the Viterbi decoder, there is a minimum accumulated distance, the minimum distance among accumulated path metrics, which tells us about channel status, for example, minimum accumulated distance value is small in good channel condition that means a large SNR value, and large in bad channel condition. Therefore if we have a table which is consisted of SNR values according to minimum accumulated distance value, then it is possible to estimate SNR value with just one table (SNR vs. minimum accumulated distance) of a little complexity. The simulation results are shown in Fig. 2. The measured minimum accumulated distance value is determined at the final stage of Viterbi decoder execution, so SNR value can be estimated after Viterbi decoder. These SNR estimates will be used at the next OFDM symbol, but this does not cause a large estimation error because of slowly varying wireless LAN's channel characteristic.

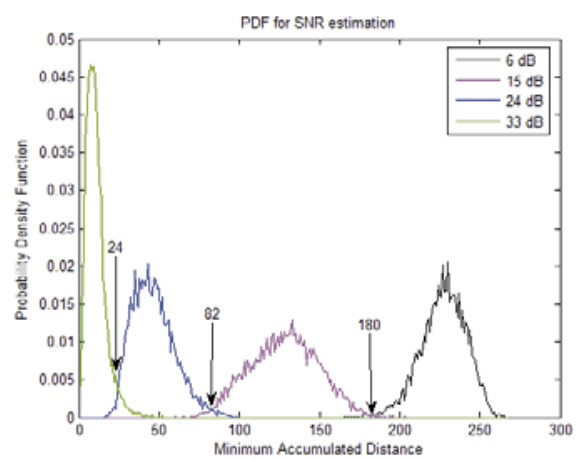

(a)

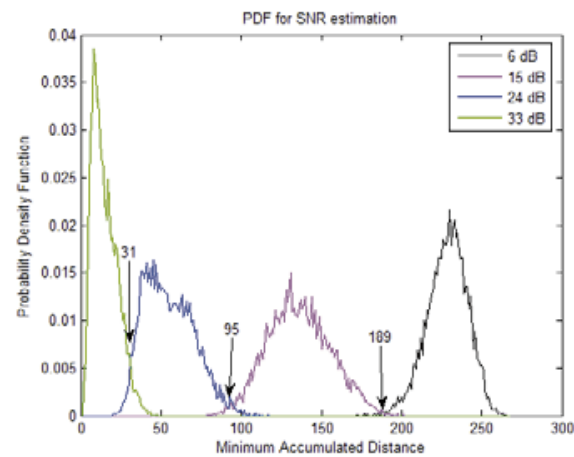

(b)

Fig. 2. Probability of Minimum Accumulated Distance (a) HIPERLAN/2 Channel A case (b) HIPERLAN/2 Channel B case

\subsection{The LS and LMMSE Channel Estimation technique}

In an OFDM system, if transmitter sends $\mathrm{X}$ vector through wireless channel $\mathrm{H}$ with AWGN W, then received vector $\mathrm{Y}$ is represented in frequency domain. 


$$
\mathrm{Y}=\mathrm{XH}+\mathrm{W}
$$

The LS channel estimates are derived in [7] as follows.

$$
\hat{\mathrm{H}}_{\mathrm{LS}}=\mathrm{X}^{-1} \mathrm{Y}
$$

The LMMSE channel estimates are derived in [4] as follows, which needs auto-covariance matrix $R_{H Y}$ and $R_{Y Y}$ value.

$$
\hat{\mathrm{H}}_{\mathrm{LMMSE}}=\mathrm{R}_{\mathrm{HY}} \mathrm{R}_{\mathrm{YY}}^{-1} \mathrm{Y}
$$

Let channel estimates $\hat{\mathrm{H}}=\mathrm{KY}$, and using orthogonal theorem in WienerHoph equation [8], Wiener filter input and error are orthogonal to each other.

$$
E\left\lfloor(\mathrm{H}-\hat{\mathrm{H}}) \mathrm{Y}^{\mathrm{H}}\right\rfloor=E\left[(\mathrm{H}-\mathrm{KY}) \mathrm{Y}^{\mathrm{H}}\right]=\mathrm{R}_{\mathrm{HY}}-\mathrm{KR}_{\mathrm{YY}}=0
$$

In [4], $\mathrm{R}_{\mathrm{HY}}$ and $\mathrm{R}_{\mathrm{YY}}$ are derived as follows.

$$
\begin{aligned}
& \mathrm{R}_{\mathrm{HY}}=\mathrm{R}_{\mathrm{HH}} \mathrm{X}^{\mathrm{H}} \\
& \mathrm{R}_{\mathrm{YY}}=\mathrm{XR}_{\mathrm{HH}} \mathrm{X}^{\mathrm{H}}+\sigma_{n}^{2} \mathrm{I} \\
& \mathrm{K}=\mathrm{R}_{\mathrm{HH}}\left(\mathrm{R}_{\mathrm{HH}}+\sigma_{n}^{2} \mathrm{I}\left(\mathrm{XX}^{\mathrm{H}}\right)^{-1}\right)^{-1} \\
& \hat{\mathrm{H}}_{\mathrm{LMMSE}}=\mathrm{R}_{\mathrm{HH}}\left(\mathrm{R}_{\mathrm{HH}}+\sigma_{n}^{2} \mathrm{I}\left(\mathrm{XX}^{\mathrm{H}}\right)^{-1}\right)^{-1} \mathrm{Y}
\end{aligned}
$$

Here $\mathrm{X}$ is assumed to be stochastic with independent and uniformly distributed constellation points. In that case, the auto-covariance matrix of the noise becomes $\mathrm{R}_{\hat{\mathrm{W}} \hat{\mathrm{W}}}=\frac{\beta}{\mathrm{SNR}} \mathrm{I}$, where $\beta=E\left\{\left|X_{k}\right|^{2}\right\} E\left\{\left|X_{k}\right|^{-2}\right\}$, in case of 16-QAM $\beta$ is calculated as $17 / 9$ and SNR is the per-symbol signal-to-noise ratio.

The LMMSE estimate now becomes

$$
\hat{\mathrm{H}}_{\mathrm{LMMSE}}=\mathrm{R}_{\mathrm{HH}}\left(\mathrm{R}_{\mathrm{HH}}+\frac{\beta}{\mathrm{SNR}} \mathrm{I}\right)^{-1} \hat{\mathrm{H}}_{\mathrm{LS}}
$$

\subsection{Distinction between HIPERLAN/2 Channel A and Channel B}

As we can see from Fig. 2, as the probability density functions of minimum accumulated distance vary according to the channel, we have to know the difference between channels. The simple method of knowing the difference is using IFFT as for the frequency response of estimated channel and decide to which channel time dispersion is close. It is a criterion whether channel is $\mathrm{A}$ or $\mathrm{B}$ that the number of measured channel taps is over 9 taps, because the maximum number of taps of channel $\mathrm{A}$ is 9 taps and 16 taps for channel B case. In case of channel A, if measured MAD value is over 180, SNR is estimated as $6 \mathrm{~dB}$, if measured MAD value is $82 \sim 179$, SNR is estimated as $15 \mathrm{~dB}$, if measured MAD value is $24 \sim 81$, SNR is estimated as $24 \mathrm{~dB}$, if measured MAD value is below $24, \mathrm{SNR}$ is estimated as $33 \mathrm{~dB}$. In case of channel $\mathrm{B}, 6 \mathrm{~dB}, 15 \mathrm{~dB}, 24 \mathrm{~dB}$ and $33 \mathrm{~dB}$ of $\mathrm{SNR}$ value is differentiated by 189,95 and 31 of MAD value. 


\section{Simulations}

HIPERLAN/2 channel model A with 9 paths and r.m.s. delay spread 68.5 nsec and channel model B with 16 paths and r.m.s. delay spread $119.1 \mathrm{nsec}$ are used for simulation. Each path experiences independent Rayleigh fading with doppler frequency of $f_{D}=50 \mathrm{~Hz}$. The parameters of the simulated OFDM system are set as follows. The entire bandwidth of $20 \mathrm{MHz}$ is divided into 64 subcarriers. The period of one OFDM symbol including effective symbol period, $T_{S}=3.2 \mu \mathrm{s}$, and guard interval, $0.8 \mu \mathrm{s}$, is $4 \mu \mathrm{s}$. For the LS channel estimation, preamble signal of long training field [1] is used. The estimated SNR value measured in Viterbi decoder may be used at the next OFDM symbol because wireless LAN channel is changing slowly. Probability density functions of minimum accumulated distance performance for several SNRs in HIPERLAN/2 channel model A and B are given in Fig. 2. As we can see, SNR difference by $9 \mathrm{~dB}$ can be differentiated by minimum accumulated distance. Mean square error and bit error rate vs. Eb/N0 performances are given in Fig. 3 respectively, we can obtain $1.8 \mathrm{~dB} \mathrm{~Eb} / \mathrm{N} 0$ gain.

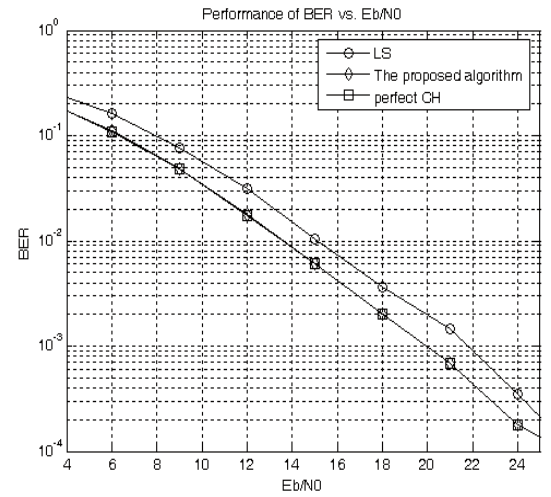

(a)

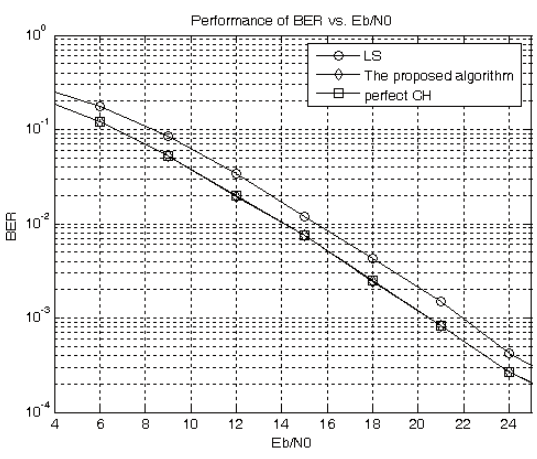

(c)

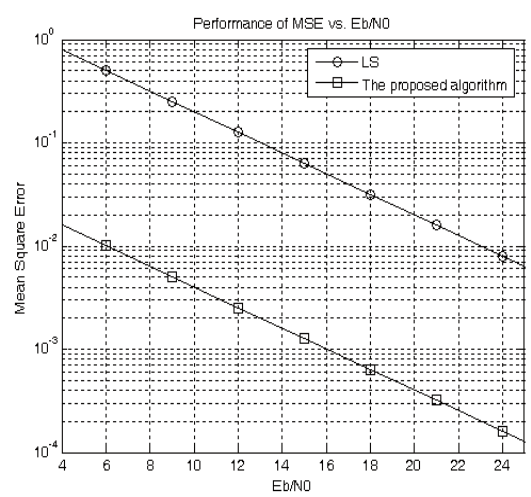

(b)

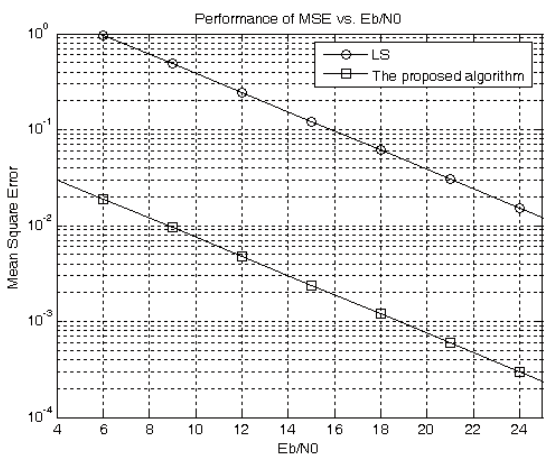

(d)

Fig. 3. Performances in HIPERLAN/2 Channel A and $\mathrm{B}$ (a) BER vs. $\mathrm{Eb} / \mathrm{NO}$ (b) MSE vs. Eb/N0 for Channel A case (c) BER vs. Eb/N0 (d) MSE vs. $\mathrm{Eb} / \mathrm{N} 0$ for Channel B case 


\section{Conclusions}

In this paper a hybrid technique which combines SNR estimation and the LMMSE channel estimation is proposed for the reduction of the implementation complexity in a receiver for low SNRs. Using minimum accumulated distance in 3 bit soft decision Viterbi decoder, we can measure SNR value by $9 \mathrm{~dB}$ interval, these values can be used in the LMMSE channel estimator. The simulation results show that with this proposed approach of a little complexity we can obtain the similar performance as the perfect channel state information and $1.8 \mathrm{~dB} \mathrm{~Eb} / \mathrm{N} 0$ gain in comparison with the LS channel estimation algorithm.

\section{Acknowledgments}

This work was supported by the second stage of BK 21 . 